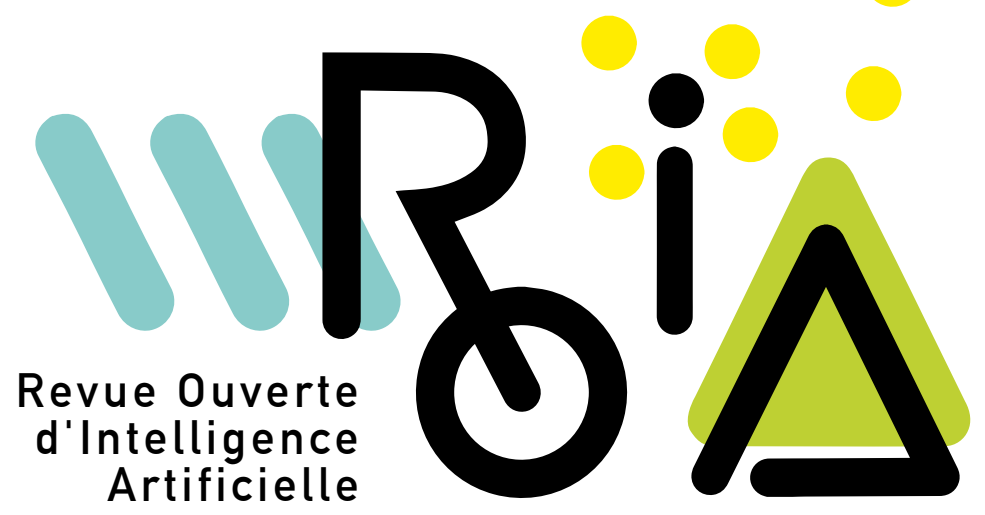

Frédérick Garçia, Thomas Guyet, Catherine Roussey Introduction ROIA Agriculture Numérique

Volume 2, no 1 (2021), p. 1.

<http://roia.centre-mersenne.org/item?id=ROIA_2021_2_1_1_0>

(C) Association pour la diffusion de la recherche francophone en intelligence artificielle et les auteurs, 2021, certains droits réservés.

\begin{tabular}{|l|l|l}
\hline (cc)BY & BY \\
\hline
\end{tabular}

Creative Commons Attribution 4.0 International License.

http://creativecommons.org/licenses/by/4.0/ 


\section{Introduction ROIA Agriculture Numérique}

Nous tenons à remercier les relectrices et relecteurs de ce numéro spécial pour leur implication :

- Florence Amardeilh, Elzeard, Bordeaux

- Olivier Boissier, LIMOS CNRS UMR 6158, Mines Saint-Etienne

- Agnès Braud, Université de Strasbourg, CNRS, ICube UMR 7357

- Antoine Cornuéjols, AgroParisTech

- Catherine Faron, Université Côte d'Azur

- Frédérick Garcia, INRAE Occitanie Toulouse, MIAT

- Thomas Guyet, Institut Agro/IRISA

- Dino Ienco, INRAE Occitanie-Montpellier, UMR TETIS

- Roland Lenain, Université Clermont Auvergne, INRAE CARA, TSCF

- Philippe Leray, LS2N/DUKe, Université Nantes

- Christopher Leturc, LIMOS CNRS UMR 6158, Mines Saint-Etienne

- Pierre Marquis, CRIL, Université Artois, CNRS, Institut Universitaire de France

- Véronique Masson Irisa, Université Rennes 1

- Engelbert Mephu Nguifo, Université Clermont Auvergne, LIMOS CNRS UMR 6158

- Pascal Neveu, INRAE Occitanie Montpellier, Mistea

- Christian Pichot, INRAE PACA, URFM

- Catherine Roussey, Université Clermont Auvergne, INRAE CARA, TSCF

- François Schwarzentruber, École normale supérieure de Rennes

- Fabien Spindler, Inria Rennes - Bretagne Atlantique

- Patrick Taillandier, INRAE Occitanie-Toulouse, MIAT / IRD, UMI UMMISCO

- Nicolas Verstaevel, Université Toulouse 1 Capitole, IRIT 